\title{
The PMS Eclipsing Binary RXJ 0529.4+0041
}

\author{
E. Covino ${ }^{1}$, S. Catalano ${ }^{2}$, A. Frasca ${ }^{2}$, E. Marilli ${ }^{2}$, J.M. Alcalá ${ }^{1}$, M. \\ Fernández ${ }^{3}$, C. Melo ${ }^{4}$, R. Paladino ${ }^{1}$, and B. Stelzer ${ }^{3}$ \\ ${ }^{1}$ Capodimonte Astronomical Observatory, Napoli, Italy \\ ${ }^{2}$ Catania Astrophysical Observatory, Italy \\ ${ }^{3}$ Max-Planck Institut für Extraterrestrische Physik, Garching, Germany \\ ${ }^{4}$ Observatoire de Genève, Sauverny, Switzerland
}

\begin{abstract}
We report the discovery of the first low-mass pre-main sequence eclipsing binary among a sample of double-lined spectroscopic binaries in the Orion star forming region found in a previous high-resolution spectroscopic investigation on ROSAT-discovered weak-T Tauri stars. Here we present the preliminary results from the combined analysis of the spectroscopic orbit and B and V light-curves, using data available till spring 2000 . We then compare the fundamental stellar parameters derived from the orbital solution with those inferred from some widely used theoretical evolutionary models.
\end{abstract}

\section{Introduction}

In the framework of a coordinated monitoring campaign to search for and study low-mass pre-main sequence (PMS) eclipsing binaries among the new doublelined spectroscopic binaries found on the basis of ROSAT optical follow-up observations in the Orion, Lupus, and Chamaeleon star forming regions (Alcalá et al. 2000; Wichmann et al. 1999; Covino et al. 1997), we report the discovery of the first low-mass PMS double-lined eclipsing binary, RXJ 0529.4+0041.

Thanks to this quite favourable circumstance, it is now possible to constrain the orbital inclination angle for this system and to obtain a direct determination of the absolute masses of the binary components, as well as of other fundamental orbital and stellar parameters (in particular, orbit size, effective temperature and stellar radius of each component), through combined analysis of the radial velocity and photometric light-curves. This information can then be used to set constraints on current PMS theoretical evolutionary models.

We emphasize that this is actually the first bona-fide PMS eclipsing binary in which both components are low-mass stars. In the other two cases known from the literature, EK Cep and TY CrA, only the secondary is a low-mass PMS star, while the more massive primary is a Herbig Ae star, already very near the ZAMS (Mathieu et al. 1999). 

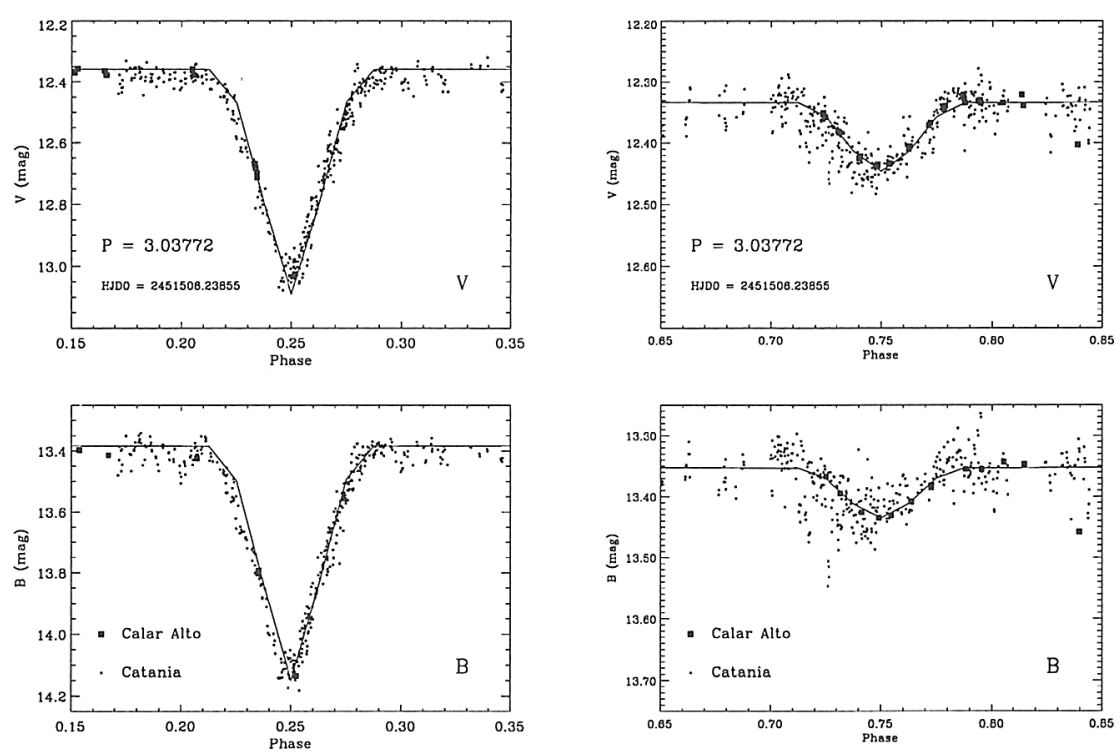

Figure 1. Photometric (B and V) light-curves folded in phase: details of the primary and secondary eclipses.

\section{Observations}

The bulk of photometric observations have been carried out during winter 19992000 with the $0.9 \mathrm{~m}$ telescope of Catania Observatory and with the Spanish $1.5 \mathrm{~m}$ telescope at Calar Alto.

The high-resolution spectroscopy was obtained during several observing seasons at the Calar Alto Observatory with FOCES at the $2.2 \mathrm{~m}$ telescope, and at ESO-La Silla with CORALIE at the $1.2 \mathrm{~m}$ Swiss telescope.

RXJ 0529.4+0041 is a well detached system, with a circular orbit and a relatively short orbital period (nearly equal to 3 days). The eclipses last just a few hours and escaped detection until November 1999, also due to insufficient precision of the early spectroscopic ephemerides. Fig. 1 shows the detail of the primary and secondary minima in the Johnson B and V bands folded in phase with the orbital period.

\section{Preliminary Orbital Solution}

The light-curve solution presented here is obtained by adopting a simple model and using as few free parameters as possible, using the data available till spring 2000. As a first guess, all parameters already known from the spectroscopicorbit solution and the effective temperatures derived from the spectral types (using the de Jager \& Niewuwenhuijzen's (1987) calibration) have been used to estimate unknown parameters (e.g., orbital inclination and fractional radii of the stars), to a first approximation. Several iterations have been performed using 
Table 1. Derived orbital and stellar parameters

\begin{tabular}{|c|c|c|c|c|}
\hline \multicolumn{2}{|c|}{ Orbital parameters } & \multicolumn{3}{|c|}{ Stellar parameters } \\
\hline & & & Primary & Secondary \\
\hline $\mathrm{P}_{\text {orb }}$ (days) & $3^{d} .03772 \pm 0.00001$ & $\mathrm{M}\left(\mathrm{M}_{\odot}\right)$ & $1.30 \pm 0.03$ & $0.95 \pm 0.03$ \\
\hline & $0.0 \pm 0.007$ & $\mathrm{SpT}$ & K1-K2 & K7-M0 \\
\hline$\gamma\left(\mathrm{km} \mathrm{s}^{-1}\right)$ & $19 \pm 0.5$ & $\mathrm{~T}_{e f f}(\mathrm{~K})$ & $5100 \pm 100$ & $4000 \pm 100$ \\
\hline$i$ & $86.5^{\circ} \pm 1$ & Roche fill-fact. & $0.4 \pm 0.05$ & $0.3 \pm 0.05$ \\
\hline $\mathrm{M}_{2} / \mathrm{M}_{1}$ & $0.73 \pm 0.02$ & $\mathrm{R}\left(\mathrm{R}_{\odot}\right)$ & $1.6 \pm 0.2$ & $1.2 \pm 0.2$ \\
\hline$a\left(\mathrm{R}_{\odot}\right)$ & $11.56 \pm 0.01$ & $\mathrm{~L}\left(\mathrm{~L}_{\odot}\right)$ & $1.6 \pm 0.15$ & $0.3 \pm 0.15$ \\
\hline
\end{tabular}

the result of the previous best-fit as a starting point and by allowing up to four parameters to vary simultaneously (namely, the orbital inclination, the effective temperature of the secondary, and the fractional radii of the components).

The derived orbital and stellar parameters are reported in Table 1. The orbital inclination, of nearly $87^{\circ}$, is not sufficient, given the separation and sizes of the two components of RXJ 0529.4+0041, for producing a total eclipse, although a major fraction of the secondary component is occulted at the secondary minimum.

\section{Evolutionary Status}

The PMS nature of RXJ 0529.4+0041 is assessed on the basis of its spatial location and kinematics, indicating that the system is a very likely member of the Orion OB1a association, as well as on the high lithium content observed in each of the individual components (Covino et al. in preparation). An independent estimate of the distance, from the observed flux and the total luminosity calculated directly using the radii and effective temperatures derived from the light-curve solution, yields a value $350 \mathrm{pc}$, in good agreement with the determination based on HIPPARCOS parallaxes for the Orion OB1a subgroup (Brown et al. 1998).

Figure $2(a$ and $b)$ shows the position on the HR diagram of the binary components with respect to the sets of theoretical evolutionary tracks and isochrones by D'Antona \& Mazzitelli (1994), and Swenson et al. (1994), respectively. Although there are obvious differences from one model to the other, the comparison indicates that the two stars are fairly coeval, within the uncertainties, with ages of about $10^{7}$ years. The primary component lies already on the radiative part of the PMS track, while the secondary falls on the final part of the corresponding convective Hayashi track. Thus, at least the primary component can be considered a 'bona-fide' post-T Tauri star.

In all cases, the mass inferred from the theoretical tracks for the primary component is consistent, within the errors, with the observational value whereas, in the case of the secondary component, a better agreement is found for the Swenson's tracks. However, from our observations, we find indication of possible out-of-eclipse variability which might especially affect the depth of the shallow secondary minimum and, hence, the determination of the effective temperature of the secondary component. RXJ $0529.3+0041$ is currently under study to 

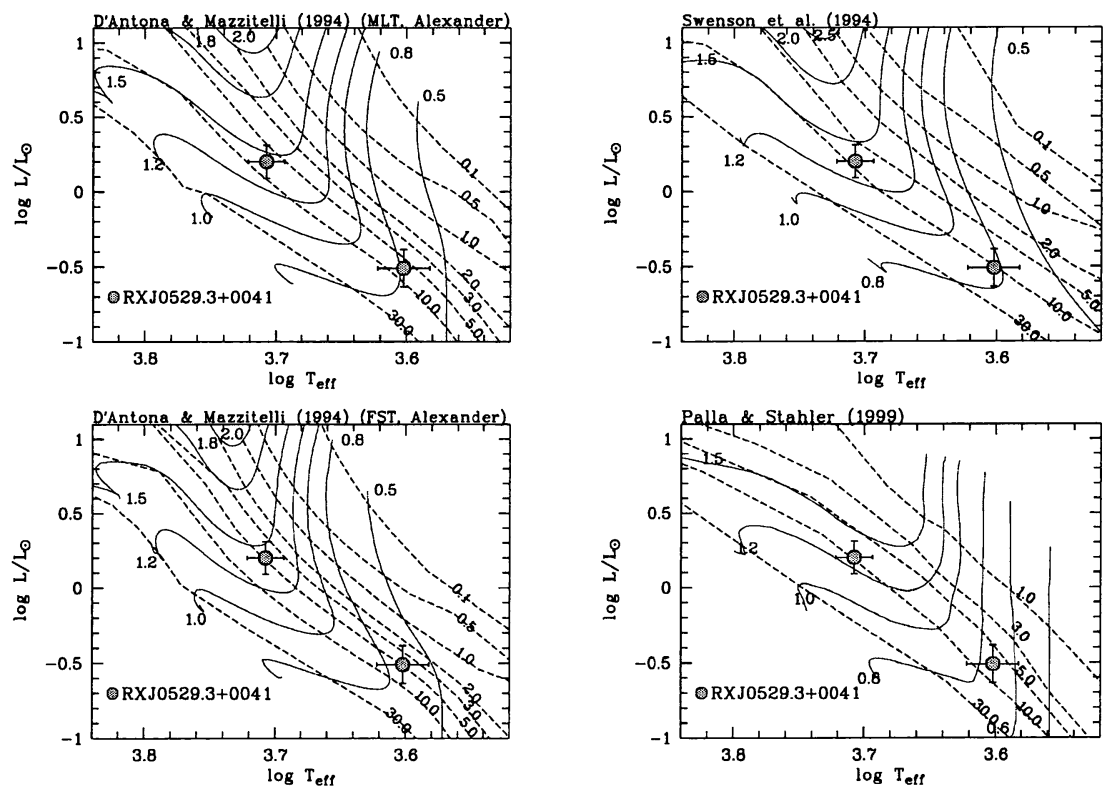

Figure 2. HR diagram for the components of RXJ 0529.3+0041. Comparison with the theoretical evolutionary tracks and isochrones by D'Antona \& Mazzitelli (1994) (left pannels), Swenson et al. (1993) and Palla \& Sthaler (1999) (upper and lower right pannels, respectively). Tracks are labelled in solar masses, isochrones in units of $10^{6}$ years.

obtain light-curves in different photometric bands, as well as to investigate in more detail the out-of-eclipse variability, in order to provide better constraints on the effective temperatures of the components.

\section{References}

Alcalá, J. M., Covino, E., Torres, G., et al. 2000, A\&A, 353, 186

Brown, A. G. A., Walter, F. M., Blaauw, A. 1998, in The Orion Complex revisited, proc. of Ringberg conference, ed. A. Burkert \& M. McCaughrean, PASP conf. Ser., in press

Covino, E., Alcalá, J. M., Allain, S., et al. 1997, A\&A, 328, 187

D’Antona, F., \& Mazzitelli, I. 1994, ApJ Suppl., 90, 467

de Jager, C., Niewuwenhuijzen, H. 1987, A\&A, 177, 217

Mathieu, R. D., Ghez, A. M., Jensen, E. L. N., Simon, M. 1999, Protostars \& Planets IV, Tucson Univ. of Arizona Press, in press

Palla, F., Stahler, S. W. 1999, ApJ, 525, 772

Swenson, F. J., Faulkner, J., Rogers, F. J., Iglesias, C. A. 1994, ApJ, 425, 286

Wichmann, R., Covino, E., Alcalá, J. M., et al. 1999, MNRAS, 307, 909

Zahn, J.-P., Bouchet, L. 1989, A\&A, 223, 112 